\section{Band 29, Heft 2, April 2013}

\section{Schwerpunkt: Hepatozelluläres Karzinom - ein interdisziplinäres Krankheitsbild \\ Herausgeber: Ricke, J. (Magdeburg); Malek, N.P. (Tübingen); Neumann, U.P. (Aachen)}

\section{Editorial}

71 Hepatozelluläres Karzinom - ein interdisziplinäres Krankheitsbild

Ricke, J. (Magdeburg); Malek, N.P. (Tübingen); Neumann, U.P (Aachen)

\section{Übersichtsarbeiten}

72 Bildgebung beim hepatozellulären Karzinom: Leitlinien und Zukunftsaussichten Bartolozzi, C.; Battaglia, V. (Pisa)

78 Histopathologie und Leberbiopsie des hepatozellulären Karzinoms

Solaß, W.; Tannapfel, A. (Bochum)

84 Systemische Therapie des hepatozellulären Karzinoms

Kirstein, M.M.; Vogel, A. (Hannover)

92 Lokoregionäre transarterielle Therapien für das hepatozelluläre Karzinom: Transarterielle Chemoembolisation und Radioembolisation Iñarrairaegui, M.; D’Avola, D.; Sangro, B. (Pamplona/Barcelona)

103 Resektion und Lebertransplantation bei hepatozellulärem Karzinom

Farkas, S.A.; Schlitt, H.J. (Regensburg)

Interdisziplinäres Gespräch

113 Hepatozelluläres Karzinom - ein interdisziplinäres Krankheitsbild

Malek, N.P. (Tübingen); Neumann, U.P. (Aachen); Ricke, J. (Magdeburg) (Gesprächsleiter)

\section{Vol. 29, Issue 2, April 2013}

\section{Focus: Hepatocellular Carcinoma - an Interdisciplinary Disease Pattern \\ Editors: Ricke, J. (Magdeburg); Malek, N.P. \\ (Tübingen); Neumann, U.P. (Aachen)}

\section{Editorial}

71 Hepatocellular Carcinoma - an Interdisciplinary Disease Pattern

Ricke, J. (Magdeburg); Malek, N.P. (Tübingen); Neumann, U.P. (Aachen)

\section{Review Articles}

72 Imaging in Hepatocellular Carcinoma: Guidelines and Future Prospects

Bartolozzi, C.; Battaglia, V. (Pisa)

78 Histopathology and Liver Biopsy of Hepatocellular Carcinoma

Solaß, W.; Tannapfel, A. (Bochum)

84 Systemic Therapy for Hepatocellular Carcinoma Kirstein, M.M.; Vogel, A. (Hannover)

92 Locoregional Transarterial Therapies for Hepatocellular Carcinoma: Transarterial Chemoembolization and Radioembolization Iñarrairaegui, M.; D’Avola, D.; Sangro, B. (Pamplona/Barcelona)

103 Resection and Liver Transplantation of Hepatocellular Carcinoma Farkas, S.A.; Schlitt, H.J. (Regensburg)

Interdisciplinary Discussion

113 Hepatocellular Carcinoma - an Interdisciplinary Disease Pattern

Malek, N.P. (Tübingen); Neumann, U.P. (Aachen); Ricke, J. (Magdeburg) (Discussion Leader) 


\section{Band 29, Heft 2, April 2013}

Fallbericht

121 Chirurgische Exploration als definitiver Weg der Diagnose einer unilateralen idiopathischen retroperitonealen Fibrose: Ein Fallbericht Troja, A.; El-Sourani, N.; Antolovic, D.; Raab, H.-R. (Oldenburg)

Originalarbeit

125 Nutzen der Alters-Albumin-Ratio als einzelner Prädiktor für die 30-Tage-Mortalität nach Kolektomie

Chin, C.-C (Chiayi, Taoyuan); Yen, C.-H. (Taipei); Hsu, W.-L. (Chiayi); Wang, J.-Y. (Chiayi, Taoyuan); Chang, C.-H. (Chiayi)

131 News / Ticker

134 Tagungen und Kongresse

136 Guidelines for Authors

119 Impressum
Vol. 29, Issue 2, April 2013

Case Report

121 Surgical Exploration as the Definitive Means of Diagnosing a Unilateral Idiopathic Retroperitoneal Fibrosis: Report of a Case

Troja, A.; El-Sourani, N.; Antolovic, D.; Raab, H.-R. (Oldenburg)

Original Article

125 Use of Age-Albumin Ratio as a Single Predictor of 30-Day Mortality after Colectomy

Chin, C.-C (Chiayi, Taoyuan); Yen, C.-H. (Taipei); Hsu, W.-L.

(Chiayi); Wang, J.-Y. (Chiayi, Taoyuan); Chang, C.-H. (Chiayi)

131 News / Ticker

134 Meetings and Conferences

136 Guidelines for Authors

119 Imprint 